\title{
Reconstitution hydrométéorologique de la crue de l'Isère de 1859
}

\author{
Annick AUFFRAY $^{1^{*}}$, Anne CLAVEL ${ }^{1 * *}$, Sylvie JOURDAIN ${ }^{1 * * *}$, Aurélien BEN DAOUD ${ }^{2}$, Eric SAUQUET ${ }^{3}$, \\ Michel $\mathrm{LANG}^{3}$, Charles OBLED 4 , Gérémy PANTHOU ${ }^{4}$, Alain GAUTHERON 5 , Frédéric GOTTARDI ${ }^{6}$, \\ Remy GARÇON ${ }^{6}$
}

\author{
${ }^{1}$ Météo-France, *Direction Interrégionale Centre-Est, avenue Louis Mouillard, 69500 Bron Aéroport, France \\ ** Centre Départemental de la Météorologie de l'Isère, 1441 rue de Piscine, 38400 St-Martin-d'Hères, France \\ *** Direction de la Climatologie, 42 avenue G. Coriolis, 31057 Toulouse, France \\ 2. Compagnie Nationale du Rhône -Direction de l'Energie - Gestion de l'Energie -2 rue André Bonin -69316 Lyon Ced 04 \\ 3. Cemagref-Unité de Recherche Hydrologie-Hydraulique, 3 bis quai Chauveau, CP 220, 69336 Lyon cedex 9, France \\ 4. Laboratoire d'étude des Transferts en Hydrologie et Environnement, Institut National Polytechnique de Grenoble, B.P. 53, 38041 Grenoble \\ cedex 9, France, e-mail : charles.obled@hmg.inpg.fr \\ 5. DDE38 - Service de Prévision des Crues Alpes Nord, 17 boulevard Joseph Vallier BP 45, 38040 Grenoble cedex 9, France \\ 6. EdF-DTG, 21 avenue de l'Europe, B.P. 41, 38040 Grenoble cedex 9, France
}

RÉSUMÉ. - La reconstitution de la séquence hydrométéorologique ayant conduit à la crue de 1'Isère des $1^{\text {er }}$ et 2 novembre 1859 a été mené en quatre étapes. La première, conduite par Météo-France, a consisté à rechercher, collecter et critiquer les données encore disponibles, notamment, les stations mesurant la pression atmosphérique en France. Cela a permis de tracer pour tout le mois d'octobre 1859 les cartes de pression au niveau de la mer, et de les interpréter en termes de circulation et de types de temps. Pour chaque journée de 1859, ces cartes ont été utilisées par le Cemagref de Lyon pour rechercher, dans la période récente $(1953-2005)$ des situations analogues $(\sim 35)$ mais dont on connaissait cette fois les précipitations sur le bassin de l'Isère, d'où une appréciation probabiliste des quantités potentiellement tombées en 1859. En utilisant ce signal, ainsi que les précipitations mesurées en 1859 en quelques stations, toutes situées en périphérie du bassin, l'INPG-LTHE a essayé de proposer un scénario vraisemblable des précipitations, mais aussi des températures, sur l'Isère et ses différents sous-bassins. Après expertise par EdF et Météo-France, ce scénario a été introduit dans un modèle Pluie-Neige-Débit par le SPC Alpes Nord. La comparaison entre débits simulés et observés à Grenoble sur le mois d'octobre 1859 a conduit à moduler encore un peu le scénario, insuffisamment contraint par les seules stations pluviométriques. Après assimilation de ces débits de crue, qu'il reproduit alors correctement, le scénario final illustre l'enchaînement et l'importance des périodes d'humectation du bassin par les pluies du 7 au 20 octobre, d'accumulation de neige à basse altitude entre le 21 et le 30 , puis son relargage sous l'effet du réchauffement et des pluies intenses des 31 octobre et $1^{\text {er }}$ novembre. C'est cette séquence exceptionnelle qui explique l'ampleur de la crue.

Mots-clés : Crue, Isère, scenario hydrométéorologique, 1859

\section{Reconstructing the hydrometeorological scenario of the 1859 flood of the Isère river}

ABSTRACT. - Reconstructing the hydrometeorological scenario that has lead to the 1859 historical flood of the Isère river has been performed in four steps. In the first one, Meteo-France has searched for, collected, and criticized old data sets still available, in particular at stations measuring the atmospheric pressure, in France or nearby. This allowed to reconstruct sea level pressure fields for the month of October 1859, and to interpret them in terms of circulation and weather types. For each single day of 1859 , this pressure map was used by Cemagref to sort out, within the recent period (1953-2008), analog situations ( 35) for which we further have the precipitations on the Isère catchment, giving therefore a probabilistic hint of what has potentially been received in 1859. Using this signal, combined with the precipitation actually observed in 1859 at a few stations, unfortunately all located outside the catchment, INPG-LTHE has proposed a likely scenario for precipitation amounts, but also for temperature, over the whole catchment and several subbasins. This scenario has gone through the expertise of EdF and MeteoFrance forecasters, and then defines inputs in a Snow-RainfallRunoff model developed by the SPC Alpes Nord. Comparisons between simulated and observed daily discharges lead to some further tuning of the scenario, insufficiently constrained by the few rain gauges available. After the assimilation of the downstream discharges, the resulting scenario reproduces well the flood peak and illustrates how important was the links between a wetting phase by the 7 to 20th October rainfalls, then the build up of a snowpack even at low elevation from the 21 to the 30th, and then its washing out by the strong warming and intense rains of October 31st and November 1 st. This is this very unusual sequence that explain the high return period of this flood.

Key-words : Flood, Isère river, hydrometeorological scenario, 1859 


\section{INTRODUCTION}

Il y a 150 ans, l'Isère inondait Grenoble et tout le Grésivaudan, au point que cette crue des $1^{\text {er }}$ et 2 novembre 1859 a été retenue comme "crue de référence » pour les aménagements en cours. Il y a donc un intérêt certain à bien comprendre ce qui s'est passé à cette occasion, et autant que possible à le quantifier... Idéalement, nous aurions souhaité faire un retour d'expérience identique à ceux réalisés pour les crues récentes (ex : crue du Gard en 2002), mais nous verrons que les données encore disponibles se sont révélées bien plus " rares » qu'aujourd'hui... Et faute d'une série dense d'informations sur le bassin, nous devrons donc nous limiter à concevoir un ou plusieurs scénarios susceptibles d'avoir conduit à cette crue. Le minimum à attendre de ceux-ci sera qu'ils soient cohérents avec toute l'information disponible, quantitative et qualitative, et qu'ils permettent d'expliquer les débits extraordinaires qui ont été observés.

L'étude a donc été décomposée en quatre parties que nous allons maintenant décrire un peu plus en détail.

\section{CONTEXTE MÉTÉOROLOGIQUE D'OCTOBRE ET NOVEMBRE 1859}

Pour préciser les conditions météorologiques qui prévalaient avant la crue, la stratégie a consisté à :

1. récupérer les données de pression, précipitations, températures, vent et état du ciel observés en France, Suisse et Italie en 1859 , comme cela avait été fait pour les crues de 1856 [1] ;

2. développer une cartographie de champs de pression quotidiens au niveau de la mer (Pmer), en différents points d'une grille de résolution $2^{\circ} 5 \times 2^{\circ} 5$ pour la période $1^{\text {er }}$ octobre - 15 novembre 1859 sur l'Ouest de l'Europe, à partir des données existantes d'un projet européen récent EMULATE [2] et des données stations récupérées ;

3. analyser et décrire les conditions météorologiques du 20 octobre au 2 novembre 1859.

Dans le cadre du projet EMULATE, on avait déjà cherché à rassembler des données historiques de pressions quotidiennes sur l'Atlantique Nord et l'Europe de 1850 à 2003 en utilisant pour cela 39 stations (en fonctionnement en 1859), dont malheureusement une seule en France ! Le projet « Isère 1859 » a poursuivi l'effort de collecte de données historiques. Des observations en quinze stations françaises supplémentaires, dont une à Grenoble, et treize stations étrangères ont finalement été rassemblées sur la période $1^{\mathrm{er}}$ octobre - 15 novembre 1859. Un exemple de documents dépouillés est donné en Figure 1.

Grâce à ces données et à une technique d'interpolation appropriée, les champs de Pmer ont été reconstruits sur toute la période. La Figure 2 illustre les cartographies ainsi obtenues les 31 octobre et $1^{\text {er }}$ novembre 1859.

Globalement, l'année 1859 a été remarquable par un été sec avec de grandes chaleurs en juillet et août, suivi par un mois de septembre encore chaud dans le Centre-Est de la France. Des fortes pluies se sont produites en mai et en octobre, en particulier le 31 octobre.

Parallèlement, nous avons rassemblé et critiqué les séries quotidiennes de précipitations et de températures à Grenoble, Lyon, Genève, Turin et Grand Saint-Bernard pour octobre et novembre 1859 , ainsi que les observations et commentaires sur le vent (direction et parfois force) ou le type de temps (pluie, brouillard, etc.). Cela a permis de reconstituer une archive numérique par station (Figure 3). Une fois toutes ces données rassemblées, les prévisionnistes de Météo-France ont pu élaborer une description détaillée, synoptique, régionale et locale, de la séquence météorologique, qui est fournie dans le rapport final du projet.

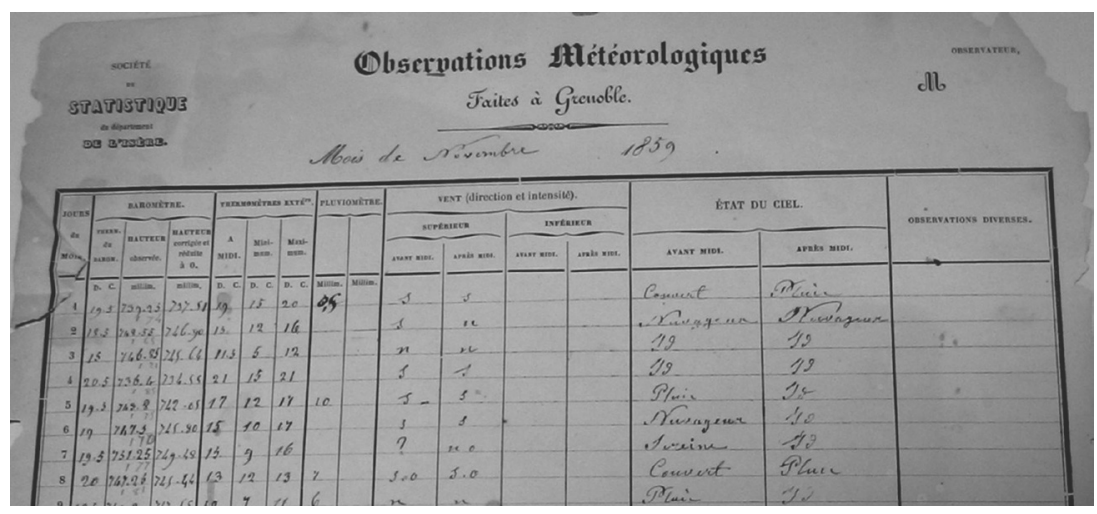

Figure 1 : Archives Météo-France-CDM38,observations à Grenoble en novembre 1859 (Photo : A. Clavel, Météo-France)
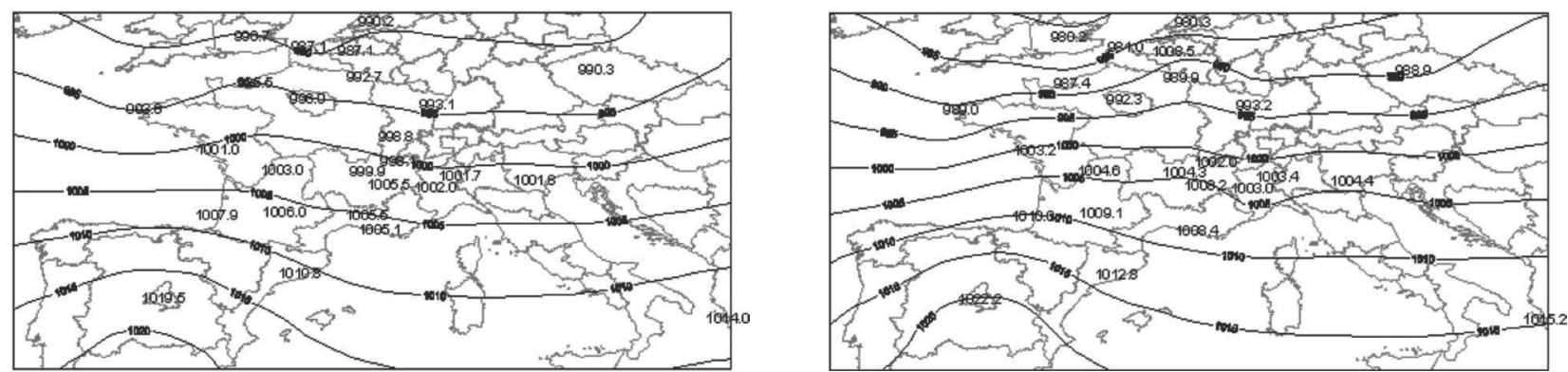

Figure 2 : Champs de pression mer Pmer sur l'Europe les 31 octobre (à gauche) et $1^{\text {er }}$ novembre 1859 (à droite) en matinée 


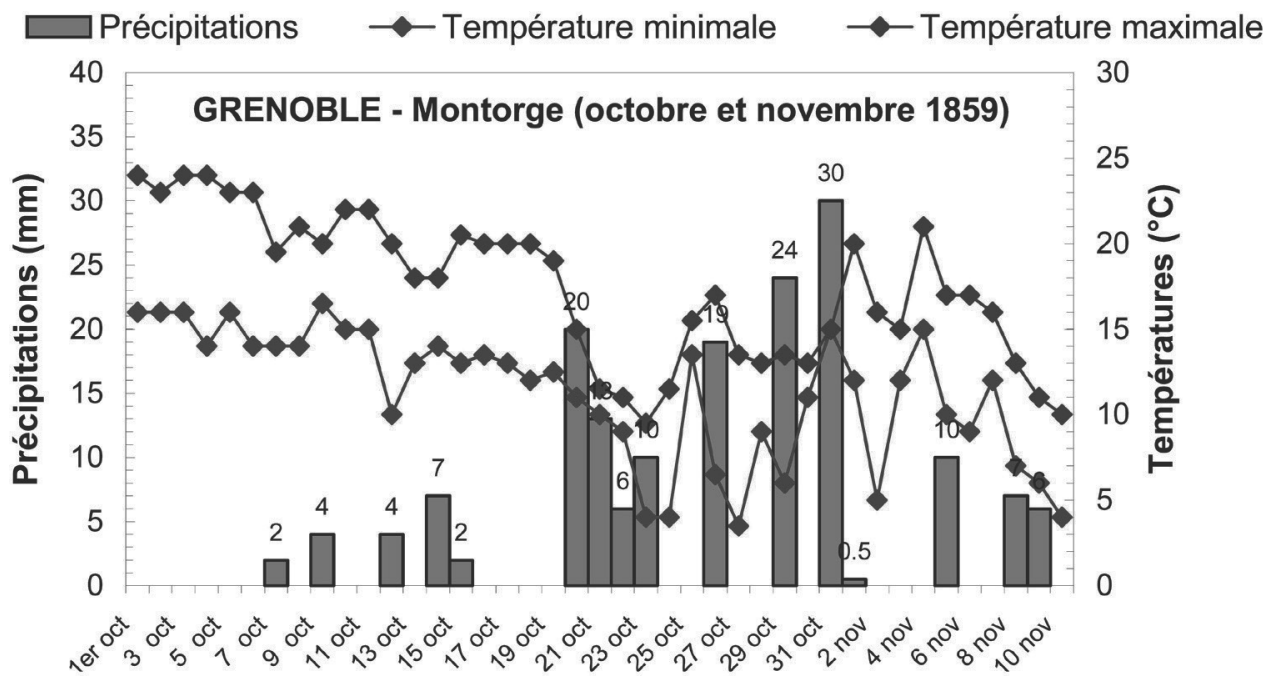

Figure 3 : Données journalières de précipitations et de températures minimale Tmin et maximale Tmax en 1859 à Grenoble

\section{RECONSTITUTION DU RISQUE NIVO- PLUVIOMÉTRIQUE

PAR UNE TECHNIQUE D'ANALOGIE

Un objectif essentiel était de reconstituer la séquence pluviométrique pendant la crue et sur toute la période la précédant, donc depuis début octobre 1859, afin de mesurer le rôle qu'elle avait pu jouer dans la saturation des terrains. En outre, cette estimation a été requise sur un découpage en sept sous-bassins (Figure 4) du bassin global de l'Isère à Grenoble $\left(4750 \mathrm{~km}^{2}\right)$, ceci afin d'évaluer la contribution respective de chacun d'entre eux à la crue.

Comme nous ne disposons que de très peu d'information sur les précipitations relevées sur le bassin de l'Isère au cours de cette période, nous avons appliqué, pour y remédier, une technique d'analogie [3]. Celle-ci permet d'identifier, dans un passé récent et bien documenté, des situations considérées, d'un point de vue météorologique, comme analogues aux situations observées en 1859. La méthode a été adaptée au bassin versant de l'Isère en amont de Grenoble et optimisée selon les données disponibles. Elle requiert en effet deux archives : une archive de situation météorologique où sont recherchées les situations analogues à la situation cible (chaque journée de 1859), et une archive pluviométrique où nous trouverons, pour ces situations, les précipitations tant aux stations que sur des sous-bassins. L'archive météorologique est constituée à partir des réanalyses NCEP-NCAR (disponibles sur 1948-2008 [4]), et une première archive pluviométrique a été élaborée à partir des réanalyses SAFRAN (disponibles seulement sur 1970-2006 au début du projet [5]).

La technique d'analogie utilisée repose sur le critère TWS de [6] permettant de mesurer la ressemblance de forme entre les champs Pmer de deux situations. Pour chaque situation journalière de 1859 , nous retenons donc les dates des situations les plus similaires entre 1970 et 2006. Sont extraites alors, pour chaque sous-bassin ou station intéressante, les pluies observées au cours de ces journées. Leur distribution empirique, ajustée par une loi Gamma, permet d'en déduire les valeurs associées à des quantiles au non-dépassement (par exemple le quantile $90 \%$, valeur qui, pour la journée

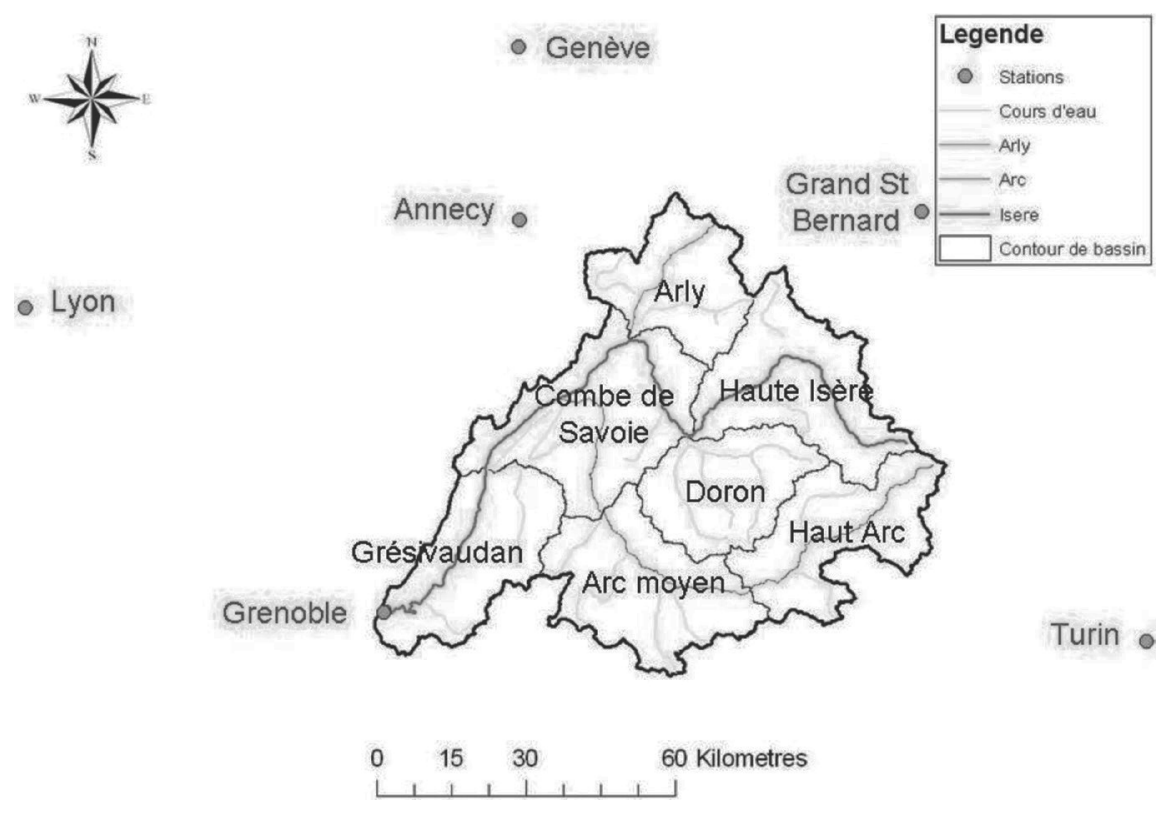

Figure 4 : Bassin de l'Isère en amont de Grenoble et sous-bassins considérés pour l'étude 

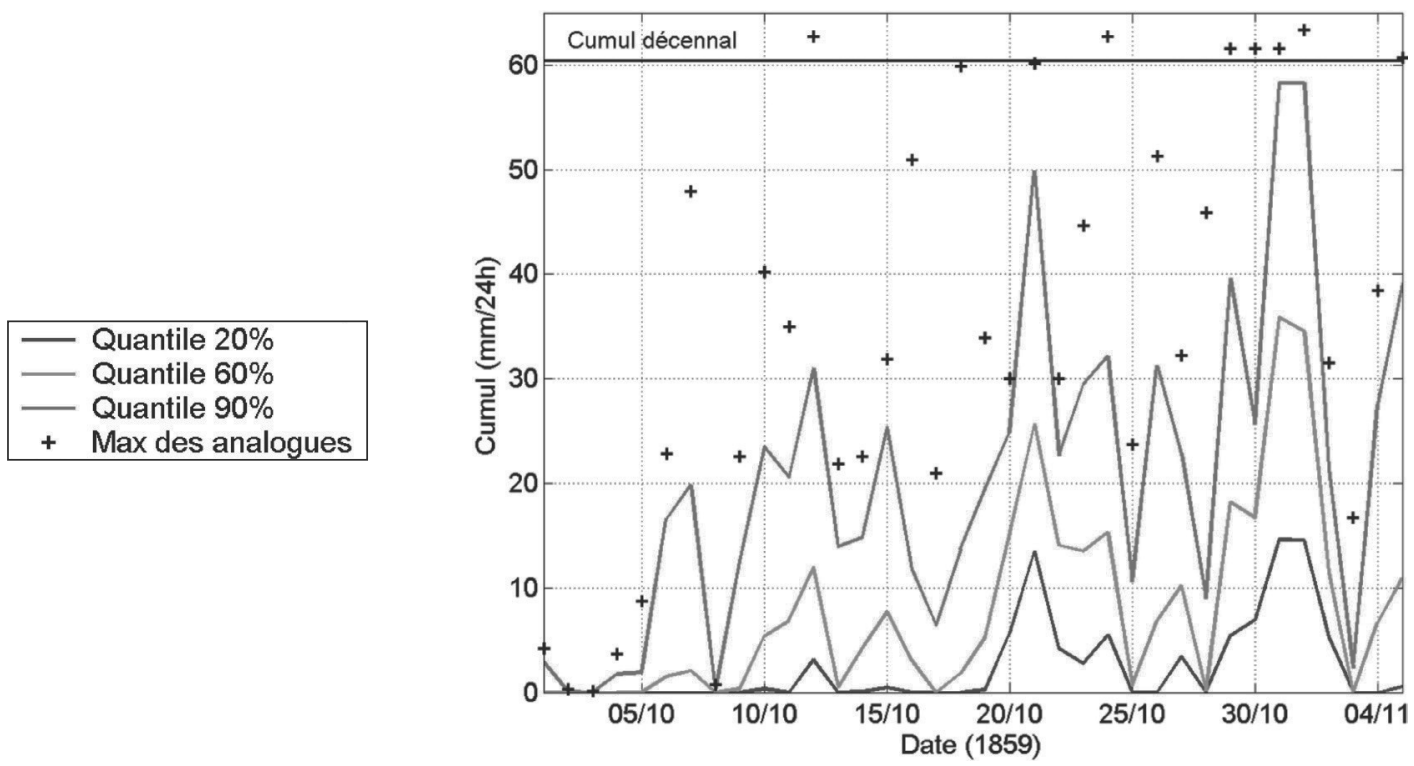

Figure 5 : Évolution des quantiles au non-dépassement prévus sur la période du 01/10 au 05/11/1859, sur le sous-bassin de l'Arly

et le bassin considéré, n'a que 10 chances sur 100 d'être dépassée).

La Figure 5 montre les courbes reliant les quantiles 20, 60 et $90 \%$, pour un sous-bassin de l'Isère, du $1^{\text {er }}$ octobre au 5 novembre 1859 . Les valeurs maximales de chaque distribution sont également indiquées afin d'avoir une idée des plus fortes précipitations observées lors des situations analogues.

Nous nous sommes intéressés aussi à la température qui aurait pu être observée à moyenne altitude. Par exemple la température au niveau de pression $850 \mathrm{hPa}$ (soit aux environs de $1500 \mathrm{~m}$ ) prise au point de grille le plus proche du bassin de $1^{\prime}$ 'Isère en amont de Grenoble $\left(5^{\circ} \mathrm{E} ; 45^{\circ} \mathrm{N}\right)$. Pour chaque journée de 1859 , les 35 analogues de la période 1970-2006 fournissent une fourchette dont l'évolution, du $1^{\text {er }}$ octobre au 5 novembre 1859 , contribuera à reconstituer les températures sur les sous-bassins. Enfin, pour chaque jour, le champ de géopotentiel $500 \mathrm{hPa}$ moyenné sur les 35 analogues donne une idée du flux d'altitude ce jour là.

Les informations issues de cette partie seront utilisées dans la section suivante en complément des précipitations et des températures observées aux stations, pour reconstruire des champs de précipitations et de températures spatialisées par sous-bassin.

\section{RECONSTITUTION D'UN SCÉNARIO DE PRÉCIPITATIONS ET DE TEMPÉRATURES}

Nous avons donc, pour chaque journée, chaque sous-bassin et chaque station, une fourchette probabiliste du « risque pluviométrique ». L'objectif suivant est alors de proposer à la modélisation hydrologique, pour chaque jour d'octobrenovembre 1859 , une valeur de précipitation moyenne sur chaque sous bassin, ainsi qu'un profil de température réaliste (en pratique l'altitude de l'isotherme $0^{\circ} \mathrm{C}$ et le gradient altitudinal de température). Cela permettra :

— de décider au dessus de quelle altitude la précipitation tombe sous forme de neige ;

- et, pour les altitudes plus basses, de calculer la lame de fonte en fonction des températures et du stock de neige disponible.
Rappelons que nous disposons :

- pour cinq stations seulement, de données de précipitations mesurées en 1859, mais dont malheureusement aucune ne se trouve dans le bassin proprement dit;

— et pour chaque journée-cible de 1859 , grâce à la méthode des analogues, d'un échantillon de journées « analogues » au moins en circulation de basses couches.

Ici, nous avons pu enrichir les résultats par analogie en ne les limitant plus à la seule période 1970-2006 dont disposait le Cemagref, mais en l'étendant à la période 1953-2005, grâce à une archive pluviométrique et thermométrique journalière sensiblement plus longue, au pas de $1 \times 1 \mathrm{~km}^{2}$, mise à notre disposition par EdF-DTG [7]. Nous avons donc pu choisir nos analogues sur une période plus longue et modifier en conséquence les fourchettes de probabilité. Mais il fallait passer d'une telle fourchette à une valeur unique pour chaque jour, constituant un scénario.

Pour cela, nous avons adopté, pour chaque journée-cible de 1859 , la méthode suivante :

- nous considérons d'abord une station, où les 35 valeurs de précipitations des journées analogues permettent de déterminer une distribution empirique, ajustée ensuite par une loi Gamma ;

- nous pouvons alors considérer la précipitation effectivement observée à la station, et en déduire sa probabilité au non dépassement dans cette distribution ;

- nous faisons alors l'hypothèse que, pour le bassin proche de la station, la probabilité de la précipitation sera la même (ou sera très voisine) de celle constatée à la station ;

- et, comme nous avons aussi la distribution attendue sur le sous-bassin pour ce jour-là, il suffit d'extraire la valeur correspondant à la même probabilité, observée sur la station, et de l'affecter au sous-bassin.

Naturellement, cette hypothèse et cette procédure ont d'abord été validées par application sur la période connue 1953-2005 pour ce qui concerne le scénario de précipitations.

Un traitement similaire a été appliqué aux températures (notamment les températures moyennes) en recherchant d'abord le niveau de l'isotherme $0^{\circ} \mathrm{C}$ à chacune des six stations, en partant de la température mesurée et d'un gradient altitudinal de température spécifique à la température 


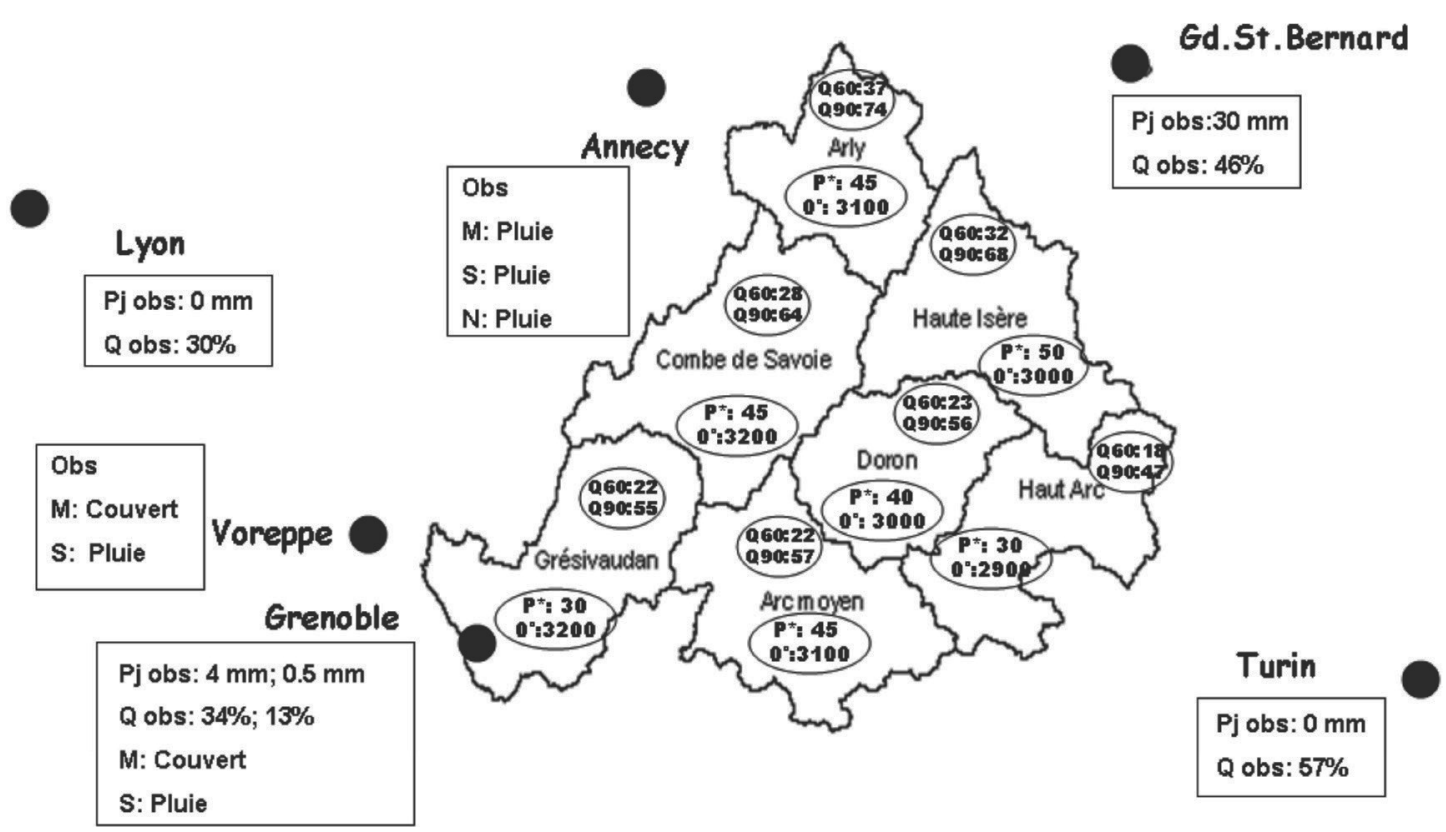

Figure 6 : Scénario II (dans les ellipses inférieures) pour la précipitation (en $\mathrm{mm}$ ) et l'altitude de l'isotherme $0^{\circ} \mathrm{C}$ (en $\mathrm{m}$ )

moyenne du mois d'octobre [7]. Ensuite, nous avons associé chaque bassin à la station la plus proche, et considéré qu'ils ont le même niveau d'isotherme $0^{\circ} \mathrm{C}$. Puis en prenant un gradient altitudinal qui cette fois est moyenné spatialement sur le sous bassin, et spécifique à la température moyenne du mois d'octobre, cela permet alors de définir, pour le sousbassin, un profil complet de température.

Pour chaque jour, et pour chaque sous-bassin (Figure 6), nous disposons ainsi d'une valeur de précipitation, une altitude d'isotherme $0^{\circ} \mathrm{C}$, et un profil de température. Nous avons reporté aussi à chaque station la donnée de pluie observée, sa probabilité dans sa distribution conditionnelle du jour, ainsi que les observations du ciel effectué le matin $(\mathrm{M})$, le soir (S) ou la nuit (N).

Ce scénario I a d'abord été soumis à expertise auprès des prévisionnistes de Météo-France, puis d'EdF, qui l'ont retouché notamment sur les journées des 31 octobre et $1^{\text {er }}$ novembre. En effet pour ces deux journées, les 2 ou 3 meilleures analogues (sur 35) sont justement des journées très fortes (12 novembre 1969, 26/27 novembre 1983) qui dépassent la décennale sur plusieurs sous-bassin. Nous pouvons donc raisonnablement augmenter sensiblement (mais sans même atteindre la décennale) la valeur affectée à ce jour là, d'où un scénario II représenté chaque jour par une carte comme celle de la Figure 6. Mais nous verrons dans la section suivante que c'est la cohérence avec les débits qui viendra encore modifier et affiner cet « ajustement».

\section{UTILISATION DE LA MODÉLISATION HYDROLOGIQUE ET DES INFORMATIONS DE DÉBITS}

Nous disposons à Grenoble de la chronique des hauteurs d'eau sur la période concernée. Différents auteurs [8] ont cherché à reconstituer une courbe de tarage valide en 1859 pour transformer ces hauteurs en débits, avec naturellement une certaine incertitude autour de l'hydrogramme reconstitué, notamment au voisinage du maximum... Ceci permet dans un premier temps de valider nos scénarios, puis de les contraindre, en regardant s'ils peuvent expliquer et permettre de reproduire raisonnablement un tel hydrogramme. Cette dernière partie a été réalisée grâce à une modélisation développée par le SPC Alpes-Nord que nous présentons succinctement dans la mesure où cette démarche a influencé fortement les résultats du projet.

Pour tenter de confirmer notre scénario, un modèle hydrologique (version adaptée du modèle conceptuel global GR4J [9]) représentant la transformation Précipitation-Neige-Débit a été utilisé. Celui-ci a d'abord été calé, en valeurs moyennes journalières, sur des années récentes pour des sous-bassins non influencés ou disposant de débits naturels reconstitués, et les paramètres ont ensuite été étendus à l'ensemble du bassin. Le modèle a été alimenté en entrée par le scénario expertisé $\mathrm{II}$ de précipitations et isothermes $0^{\circ} \mathrm{C}$. Nous avons rapidement conclu à un manque d'eau en entrée, de l'ordre d'une trentaine de millimètres sur les deux jours du 31 octobre et $1^{\text {er }}$ novembre. En les ajoutant « brutalement » sur la valeur du $1^{\text {er }}$ novembre, nous constatons d'ailleurs que cela améliore considérablement la reproduction de la crue.

Or en reconsidérant les meilleures analogues, notamment celles du 12 novembre 1969, qui avait pratiquement les mêmes valeurs aux stations qu'en 1859, et celles des 26/27 novembre 1983, nous constatons qu'elles présentent des précipitations bien plus fortes en montagne (avec un vent fort renforçant l'effet orographique, comme ce fut le cas aussi en février 1990). Ceci nous a donc incités à relever encore un peu les valeurs de certains sous bassins sur les 31 octobre et $1^{\text {er }}$ novembre 1859 . 


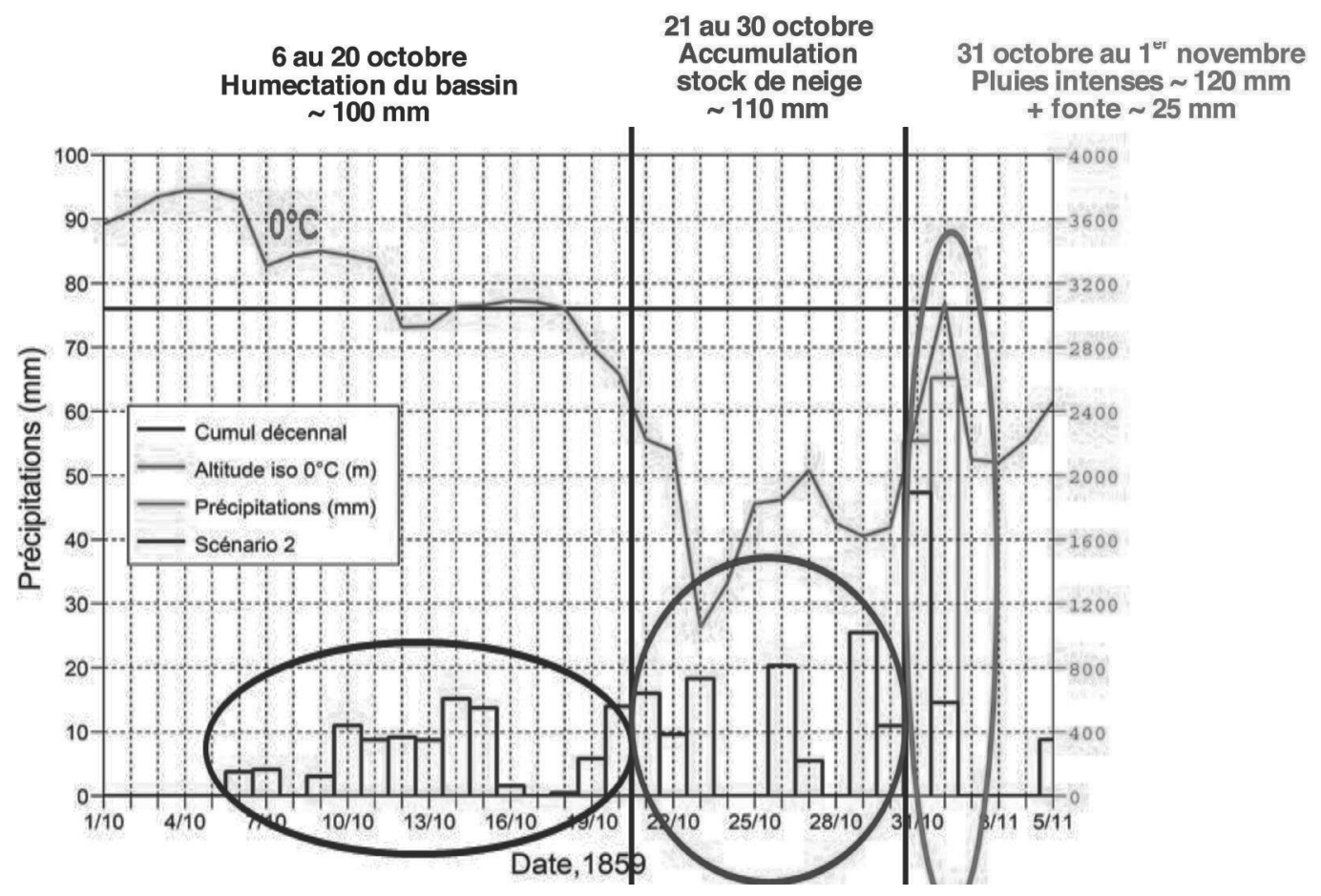

Figure 7 : Scénario III (avec en vert, les précipitations ajoutées par rapport au scénario II)

Toutefois, ce n'est pas la seule façon d'amener une lame d'eau supplémentaire ces jours là (en augmentant les précipitations à un niveau raisonnable, qui reste proche de la décennale, un peu plus sur le Doron...). Il est possible également de modifier les isothermes $0^{\circ} \mathrm{C}$ (en utilisant plutôt la Tmin que la Tmoy pour décider de la limite inférieure de l'enneigement). Ceci a pour conséquence pour la période 21-30 octobre, de générer un enneigement à plus basse altitude (1600 plutôt que $1800 \mathrm{~m}$ ), plus conforme aux observations disponibles, et de conserver la neige disponible pour fondre ultérieurement lors du réchauffement du 31 octobre, quantité qui viendra alors s'ajouter à la pluie et contribuer à la pointe.

Globalement, les modifications ont conduit au scénario III (Figure 7) qui donne en débits moyens journaliers à Grenoble des résultats très satisfaisants (Figure 8 ). Cependant, il ne s'agit plus désormais d'une validation, mais d'une assimilation d'information nouvelle à respecter (i.e. les débits à
Grenoble déduits des hauteurs et de la courbe de tarage) à travers le modèle hydrologique retenu.

Le modèle hydrologique permet aussi de proposer d'autres sous-produits, comme l'évolution du manteau neigeux (présentée en Figure 9 par tranche de $1000 \mathrm{~m}$ pour le bassin global) ou de l'état de saturation du sol, qui ne fait que croître depuis le début octobre jusqu'à la crue proprement dite.

\section{CONCLUSION}

Nous rappellerons encore, et nous insisterons sur le fait qu'il ne s'agit que de scénarios, dont nous pourrions imaginer d'autres variantes tout aussi plausibles. Ceux-ci ne peuvent être complètement validés sur des données indépendantes, d'abord parce que toutes les données ont été utilisées et qu'il y a eu de nombreuses rétroactions entre partenai-

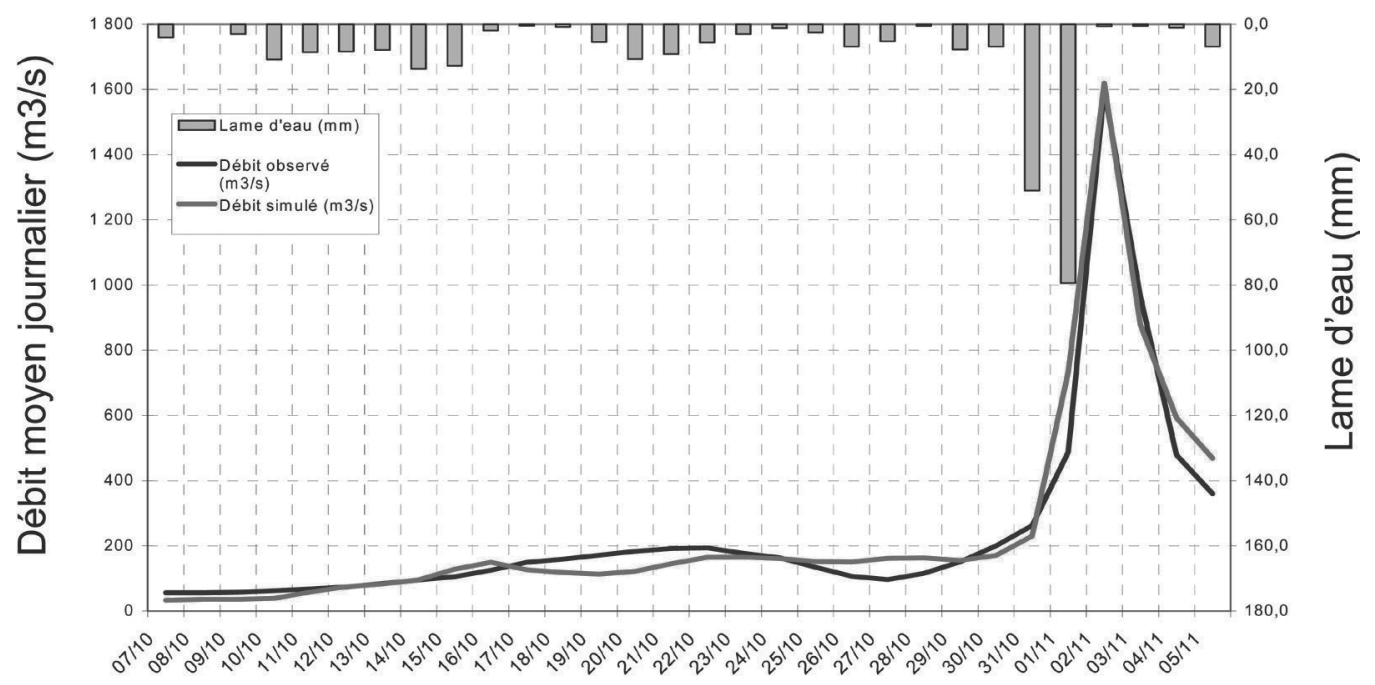

Figure 8 : Reconstitution de la crue avec le scénario III [10] 


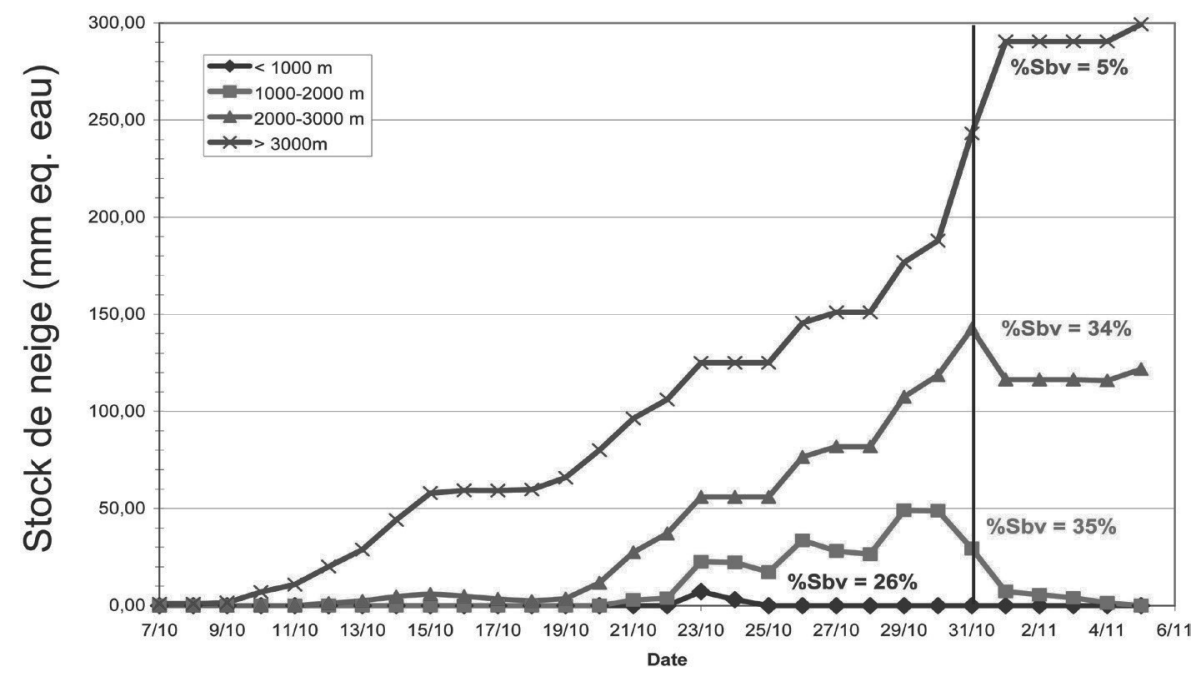

Figure 9 : Evolution du stock de neige par bande d'altitude de $1000 \mathrm{~m}$ sur le bassin global [10]

res, mais aussi parce que ces scénarios ont été construits et modifiés pour permettre justement une reconstitution correcte des débits estimés (avec un modèle donné...). De plus, compte tenu des incertitudes associées aux données mais aussi et surtout de leur médiocre couverture spatiale du bassin concerné, il est difficile de contraindre nos scénarios vers un scénario unique. L'appel à une contribution significative de la fonte de neige semble déterminant, mais pas forcément exceptionnel dans les deux jours qui ont précédé la crue. De même, nous constatons que l'épisode n'a pas « besoin » (pour reconstituer la crue !) d'avoir été exceptionnel en précipitations le jour et la veille de l'évènement :

- les cumuls en 2 jours reçus pour la totalité du bassin pour la période 31 octobre - $1^{\text {er }}$ novembre n'atteignent pas, même après renforcement du $1^{\text {er }}$ novembre dans les scénarios II et III le quantile décennal ;

- en revanche, en y ajoutant l'équivalent de 20 à $30 \mathrm{~mm}$ de fonte sur la période 31 octobre et $1^{\text {er }}$ novembre, la lame d'eau résultante sur 2 jours devient équivalente à une pluie de période de retour de 25 à 30 ans (voire un peu plus sur le Doron).

C'est donc plutôt la forte humectation du bassin par les épisodes antérieurs (5 au 20 octobre, puis du 21 au 30 octobre, avec à chaque fois des cumuls $\sim 100 \mathrm{~mm}$ ) qui explique, combinée peut-être avec l'état de dégradation du bassin, le fort rendement en débit du dernier épisode (31 octobre et $1^{\text {er }}$ novembre) et le fait que l'on passe d'une pluie à peine décennale et, avec la fonte, d'une lame d'eau à peine trentennale à une crue plus que centennale... C'est en tout cas ce que suggère la modélisation hydrologique qui cumule et combine ces différents effets depuis le début du mois d'octobre.

\section{REMERCIEMENTS}

Ce travail a été coordonné par le laboratoire LTHE de l'INP-Grenoble, avec la participation de Météo-France (CDM Grenoble, DIRCE Lyon et DCLIM Toulouse) et du Cemagref (Lyon) grâce à un financement du Conseil Général de l'Isère attribué par le Pôle Grenoblois Risques Naturels, que nous remercions. Il a bénéficié de l'expertise mais aussi des concours financiers exceptionnels d'EdF-DTG et du Service de Prévision des Crues-Alpes Nord, DDEA 38.

Les auteurs tiennent à remercier pour leur aide les services d'archives départementales de l'Isère, de la Savoie et la Haute-Savoie, et la bibliothèque dauphinoise, sans oublier bien sûr le service de climatologie de MétéoSuisse à Genève. L. Mercalli président de la Société Météorologique Italienne, D. Cœur historien et $\mathrm{S}$. Gache ont mis gracieusement à notre disposition des relevés respectivement à Genève, Turin et Grenoble et Annecy. Enfin plusieurs stagiaires ont contribué à ces travaux, dont notamment $\mathrm{G}$. Panthou, qui en fut véritablement, du côté LTHE, la cheville ouvrière.

\section{RÉFÉRENCES}

[1] Moisselin J.M., \& Jourdain S. (2007) - Recherche et valorisation de données météorologiques anciennes, La Houille Blanche. 4 33-38

[2] Ansell T.J. Et Al. (2006) - Daily mean sea level pressure reconstructions for the European - North Atlantic region for the period 1850-2003, Journal of Climate. 19 2717-2742

[3] Ben Daoud A., Sauquet E., Lang M., Bontron G., \& Obled C. (2009) - La prévision des précipitations par recherche d'analogues : état de l'art et perspectives, La Houille Blanche. 6 60-69

[4] Kalnay E. Et Al. (1996) - The NCEP/NCAR 40-Year Reanalysis Project, Bull. Amer. Meteor. Soc. 77 437-471

[5] Vidal J.P., Martin E., Franchisteguy L., Baillon M., \& Soubeyroux J.M. (2009) - A 50-year high-resolution atmospheric reanalysis over France with the Safran system. DOI 10.1002/joc.2003, Int. J. Climatol.

[6] Teweles J., \& Wobus H. (1954) - Verification of prognosis charts, Bull. Amer. Meteor. Soc. 35 455-463

[7] GotTARdi F. (2009) - Estimation statistique et réanalyse des précipitations en montagne. Utilisation d'ébauches par types de temps et assimilation de données d'enneigement. Application aux grands massifs montagneux français. Thèse de doctorat de l'INP-Grenoble. 261 pages

[8] Dumas D. (2004) - Les deux crues mémorables de l'Isère à Grenoble (1651 et 1859) : Analyse des estimations de M. Pardé, Revue de Géographie Alpine. 92 27-49

[9] Perrin C. (2002) - Vers une amélioration d'un modèle global pluie-débit au travers d'une approche comparative, La Houille Blanche. 6/7 84-91

[10] Gautheron A., Balmand E., \& Valery A. (2009) Modélisation hydrologique de la crue de l'Isère de 1859 , Colloque Crue Isère 1859-2009 (Grenoble, France, 5 novembre 2009). 25 pages 